\title{
Sound Mixing and Recording on Mobile Devices: Study of Noise Generation depending on different Parameters
}

\author{
Gaurang Mhatre \\ Student \\ Sardar Patel Institute \\ of Technology \\ Mumbai, India
}

\author{
Kunal Salunke \\ Student \\ Sardar Patel Institute of \\ Technology \\ Mumbai, India
}

\author{
Ruchir Raut \\ Student \\ Sardar patel Institute of \\ Technology \\ Mumbai, India
}

\author{
Anand Godbole \\ Asst. Professor \\ Sardar Patel Institute \\ of Technology \\ Mumbai, India
}

\begin{abstract}
Traditional and present methods for recording and mixing music are working quite well but they do not take responsibility in empowering the individuals who lack the resources for composing and recording music. In this paper combination of different approaches are taken into consideration while analyzing the performance of the mobile device while recording the sound and embedding it with background music.
\end{abstract}

\section{Keywords}

Audio, Background track, Surround Sound, Multi-threading

\section{INTRODUCTION}

In early days, mobile phone was just a communication device which connects wirelessly to the public switched telephone network. But after the launch of mobile OS such as android, iOS, Symbian the sole purpose of this hand held device is redefined [1]. Modern mobile operating systems incorporate the features of a personal computer operating system with other features, including a voice recorder, touchscreen, Bluetooth, Wi$\mathrm{Fi}$, camera, video camera, GPS mobile navigation, speech recognition, music player, near field communication and infrared blaster.

Audio recording is classified and segmented into basic audio types such as silence, music, song, speech, environmental sound, speech with the music background, environmental sounds with the music background etc. Audio, which includes voice, music, and various kinds of environmental sounds, is an important type of media and also a significant part of multimedia data [2].

To record music acquiring appropriate equipment's such as Amplifiers, Microphones, Mixers, Electric instruments is necessary for a professional singer. But this equipment's are not mandatory for a naïve individual for composing a music track in his voice. Mobile phones are the most common devices which are preloaded with the hardware and processing capability which can substitute this equipment's.

Proposed application can use hardware utilities and with simultaneously running media player and recorder it can compose new audio file with the help of multi-threading[3]. This resulting file can be stored on the native storage such as memory card or else it is uploaded to cloud storage.

\section{RELATED WORK}

Today, many research projects has main goal of giving the utilities to the individual to compose the music bypassing all the formal procedure before initiating. This can be done using mobile phones with relative ease.

In this Paper analysis of different factors such as surround sound, speaker quality and background sound playing mode are taken into consideration while recording music on a mobile device. In addition to this noise generation is also observed for different scenarios. The resulting data is combined to derive a meaningful conclusion.

\section{METHODOLOGY}

The approach to record and analyze the performance takes place in five steps which are demonstrated in figure 1.

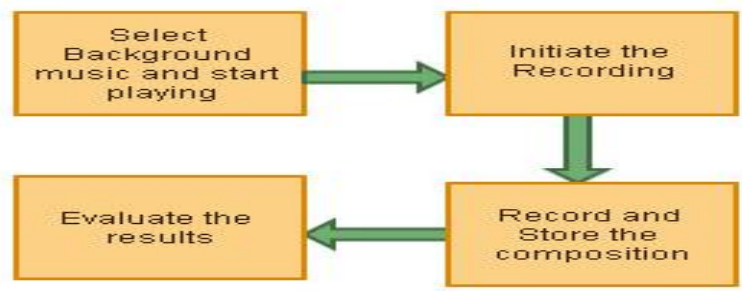

Fig 1: Flow of approach to detect performance of the system

\subsection{Selection of background music}

For the composition of new track background audio is selected. This file can be of any lossless or lossy audio file type such as .flac, .mp3, .vorbis, .wma etc [4]. For lucid analysis of the results background tracks is selected with pitches at identical intervals. Noise can identified with any asymmetry present in the end product.

\subsection{Start playing background music and activate recorder}

Once the appropriate background music is selected the music player is initiated which will play this track. This procedure is handled by a single thread running on the android mobile. Another thread is responsible for the starting the recorder and storing the product in application temporary buffer. The figure 2 shows the code for this multi-threading on android application. 


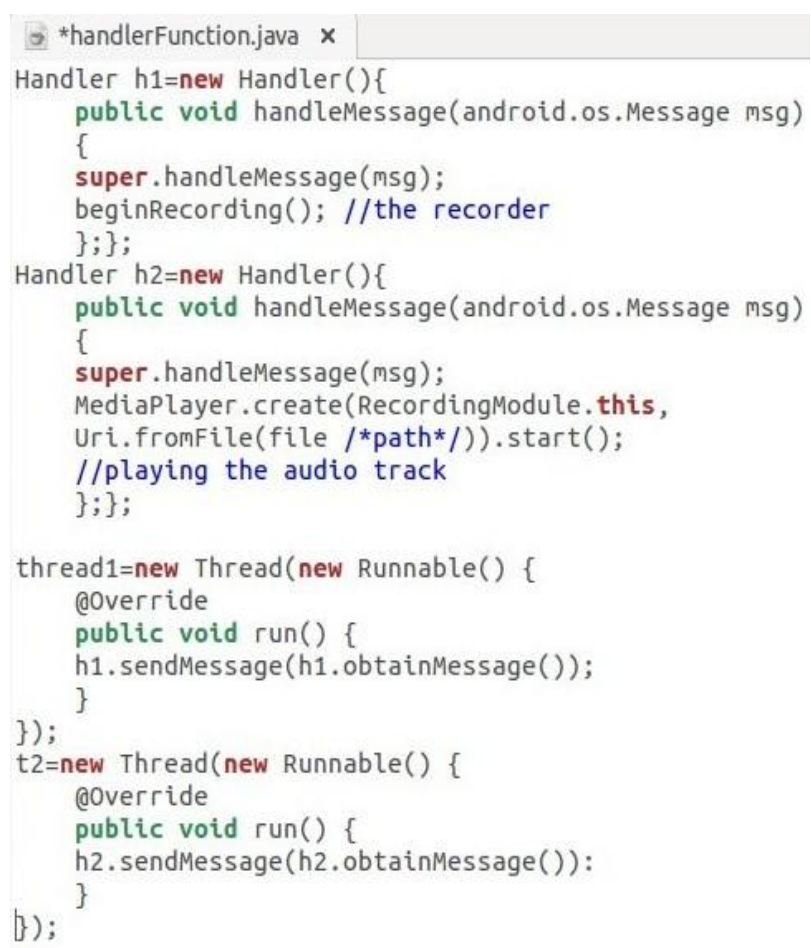

Fig 2: Java code for multi-threading between recorder and music player on android device

Two different approaches are analyzed for changes in the behavior of the resulting product.

\section{- Approach One}

In this first approach selected background Music is played via primary speaker present on the backside of the phone. The quality of the background music in the resulting product is directly proportional to the quality of the speaker mobile has.

\section{- $\quad$ Approach Two}

In this approach background music is played via headphone or else they are disabled and directly added to the recording at runtime. This helps the recorder to get rid of noise generated by low quality speakers. Also all mobile devices tend to give universal results in this approach.

\subsection{Recording the Composition}

Resulting music record is stored on the mobiles external storage card as .mp3. This Music track is analyzed for noise existence depending on the approach of playing the background music.

\subsection{Evaluating the Results}

Results are calculated in two of different scenarios around the recording. The resulting data is organized in the table shown in figure 3 . The results are evaluated with the ground trust data that is manually collected. Noise is calculated with equation given bellow.

\section{RESULTS}

The results are evaluated with the ground track data that is manually collected. Noise is calculated with equation given bellow.

Noise $(\%)=\left(\sum \#\right.$ output amplitude- $\sum \#$ input $\sum \#$ output amplitude * 100 .

amplitude) /

This noise in systems is a product of both internal and external sources to the system. By experimenting with three different parameters such as Surround sound, Background Music via speakers and Background music via headphones external noise can be reduced drastically. As shown in table in figure 3 application performs best in surround sound lower then $60 \mathrm{~dB}$ and direct embedding of background track and recording without any broadcast via speakers.

\begin{tabular}{l|ll|}
\hline & $\begin{array}{l}\text { Noise distortion } \\
\text { Background Music } \\
\text { playing via } \\
\text { speaker }\end{array}$ & $\begin{array}{l}\text { Noise distortion } \\
\text { Background Music } \\
\text { playingvia } \\
\text { headphones }\end{array}$ \\
\hline $\begin{array}{l}\text { Surround sound } \\
\text { above 60dB } \\
\text { Surround sound } \\
\text { 60dB } \\
\text { Surround sound } \\
\text { bellow 60dB }\end{array}$ & $96 \%$ & $90 \%$ \\
\hline
\end{tabular}

Fig 3: Table showing the percentage Noise present in the resulting track with respect to Surround Sound and Background Music

\section{CONCLUSION}

With simple approach and implementation any mobile phone which is capable of running multiple threads can compose good quality audio recording considering the surrounding environment and device recorder. This will empower novice individuals for singing and recording whenever they want and where ever they want.

\section{REFERENCES}

[1] Thom Holwerda, OSNews, 12 November 2013, The second operating system hiding in every mobile phone

[2] Z Liu, Y Wang, T Chen, 1997, Audio feature extraction and analysis for scene classification, in Proceedings of IEEE 1st Multimedia Workshop.

[3] Martin C. Rinard, July 2001, Analysis of Multithreaded Programs

[4] Myongsu Park, Compare Music File Formats, University of Texas Austin 